\title{
Componente laboral y su inserción en el mercado de trabajo de los graduados de la carrera de marketing y gestión de negocios.
}

\section{Labor component and its insertion in the labor market of the graduates of the career of marketing and business management.}

Ing. Luis Ángel Salazar López ${ }^{1}$, Ing. Jorge Javier Plaza Guzmán², M.Sc Efraín Velasteguí López³, Sr. Andrés Miguel Salazar L. ${ }^{4}$, Tatiana Carrasco Ruano. ${ }^{5}$

Recibido: 02-09-2017 / Revisado: 11-11-2017 Aceptado: 14-12-2017/ Publicado: 01-01-2018

\begin{abstract}
.
DOI: https://doi.org/10.33262/cienciadigital.v2i1.10

The model of higher education in the context is developed based on the theoreticalpractical orientation that is given to university students so that they can respond to the real problems of organizations, which need agile responses to situations that they are presented to the daily life of their activities, remembering that we have internal and external situations in the institutions that experience can solve.

Observing the behavior of higher education institutions and public and private organizations, it is determined that the needs are frequently changing, it is worth emphasizing that the way in which they can be linked to the workers in the labor market are the tools Strategies, that for this can execute the knowledge learned in a practical way in organizations, one of the best ways is to take them to practice academic training (Pre-professional practices), this being the key tool for students to transform their theoretical knowledge to practical

The organizations of the different productive sectors request Human Talent with experience in certain areas for the different occupational positions, the university students will not be able to integrate into the labor market with what they learned in the classrooms, because the companies need collaborators to make decisions in the reality of the context in which they operate.
\end{abstract}

${ }^{1}$ Universidad Técnica de Ambato, Ambato, Ecuador, la.salazar@uta.edu.ec

${ }^{2}$ Universidad Técnica de Machala, Machala, Ecuador, jplaza@utmachala.edu.ec

${ }^{3}$ Universidad Técnica de Ambato, Ambato, Ecuador, le.velastegui @uta.edu.ec

${ }^{4}$ Universidad Estatal de Guayaquil, Guayaquil, Ecuador, amsl-96@hotmail.com

${ }^{5}$ Universidad Internacional de La Rioja Logroño, España, tcarrascor@yahoo.com 
Keywords: Pre-Professional Practices, University, Labor Market, Skills, Professional Training.

\section{Resumen.}

El modelo de educación superior en el contexto se desenvuelve en base a la orientación teoría - práctica que se brinda a los estudiantes universitarios con el fin de que estos puedan responder a la problemática real de las organizaciones, las cuales necesitan respuestas agiles a las situaciones que se les presentan al diario vivir de sus actividades, recordando que tenemos situaciones internas y externas en las instituciones que la experiencia lo puede resolver.

Observando el desenvolvimiento de las instituciones de educación superior y las organizaciones públicas y privadas, se determina que las necesidades son frecuente mente cambiantes, vale recalcar que la forma en la cual se puede vincular a los dicentes en el mercado trabajo se ven encaminadas las herramientas o estrategias, que para ello puedan ejecutar los conocimientos aprendidos de manera practica en las organizaciones, una de las formas idóneas es llevarlos a que realicen prácticas de formación académica (Practicas pre profesionales), siendo esta la herramienta clave para que los estudiantes transformen sus conocimientos teóricos a prácticos.

Las organizaciones de los diferentes sectores productivos solicitan Talento Humano con experiencia en ciertas áreas para los diferentes puestos ocupacionales, el estudiantes universitario no podrá integrarse al mercado de trabajo con lo aprendido a las aulas, debido a que las empresas necesitan colaboradores que tomen decisiones en la realidad del contexto en el que se desenvuelven.

Palabras Claves: Practicas Pre Profesionales, Universidad, Mercado Laboral, Competencias, Formación Profesional.

\section{Introducción.}

Las practicas pre profesionales como herramienta que ayude a los universitarios en su inserción al mercado laboral en el contexto regional. Realidad de la Carrera de Marketing y Gestión de Negocios de la Facultad de Ciencias Administrativas (Universidad Técnica de Ambato). Ecuador.

En el entorno actual los medios productivos y metodologías para encontrar una oportunidad de laboral en las instituciones es limitada por el nivel de experiencia que cumplen los estudiantes universitarios, aun mas cuando las carreras de ámbitos sociales requieren de experiencia para la toma de decisiones, es fundamental adquirir conocimientos para desenvolverse en el mercado de trabajo, los dicentes que están a punto de graduarse y los nuevos profesionales de la Facultad de Ciencias Administrativas de la Universidad Técnica 
de Ambato de Ecuador, deben competir con colegas que tienen una misma rama de estudios, siendo este un medio difícil para insertarse en el contexto laboral del país.

Sin embargo la preocupación de los países es latente en cuestión de inserción laboral en todo aspecto y más aún cuando se trata de profesionales universitarios que cumplen los perfiles y requisitos para cubrir las necesidades de los sectores socio productivos (García Blanco \& Cárdenas Sempértegui, 2016), de la misma manera los modelos de inserción laboral se determinan por los perfiles ocupacionales que abarcan los contextos en los cuales se desenvuelven los estudiantes universitarios, de esta manera se podría determinar que la inserción laboral al ser un problema de todos los actores de una sociedad los estudiantes universitarios tienen un incremento de posibilidades para ingresar al mercado laboral (Vacchiano, Martí, Yepes , \& Miquel, 2018).

El componente de las competencias labores para los estudiantes universitarios parte desde las instituciones de educación superior como un pilar fundamental para minimizar los riesgos de no conseguir trabajo, lo cual en base a las acotaciones anteriormente el equipo investigación concluye que las instituciones de educación superior tienen responsabilidad con los graduados universitarios para su inserción laboral, por lo cual el nivel de competencia en mercado de trabajo se detalla en grama medida por la gestión y metodología de formación, capacitación y desarrollo de competencias de los estudiantes universitarios.

\section{FUNDAMENTOS TEÓRICOS}

En los últimos años los estudiantes universitarios se han visto forzados a ingresar al mercado de trabajo en cargos o puestos que no cumplen con su perfil profesional, una de las razones es que en toda su vida académica no adquirió experiencia en el ámbito laboral que permita el empleo adecuado 'para con los profesionales recién graduados, por lo antes mencionado existen índices de desempleo y subempleo lo cual no es adecuado para el contexto productivo de los países.

Las universidades deben ofertar al sector productivo profesionales que puedan ser competitivos en el mercado de trabajo, con competencias suficientes para lo cual fueron orientados en cada una de las asignaturas que recibieron, muchas veces se vuelven las universidad orientadoras de teorías que simulan el contexto laboral, pero sin embargo no es la realidad en la cual deben prestar sus servicios los graduados universitarios, una de las situaciones actuales es que las herramientas para integrar a los estudiantes en la vida laboral son los mecanismos de vinculación con la sociedad que tienen estas instituciones, en el contexto nacional lo denominan como practicas pre profesionales que se estipula en los órganos reguladores de los Institutos de Educación Superior.

Acotando a lo que menciona la Ley Orgánica de Educación Superior creada por la Asamblea Nacional Cosntituyente de la Republica del Ecuador (2010, pág. 37) el cual manifiesta lo siguiente con respecto a las prácticas pre profesionales: 
Art. 87 Requisitos previos a la obtención del título.- Como requisito previo a la obtención del título, los y las estudiantes deberán acreditar servicios a la comunidad mediante prácticas o pasantías pre profesionales. Debidamente monitoreadas. En los campos de su especialidad, de conformidad con los linchamientos generales definidos por el Consejo de Educación Superior. Dichas actividades se realizarán en coordinación con organizaciones comunitarias, empresas e instituciones públicas y privadas relacionadas con la respectiva especialidad.

Según el Reglamento de Régimen Académico existen dos mecanismos idóneos y legales para que el estudiante universitario obtenga experiencia y competencia en su ámbito laboral en su artículo 88 de termina que las prácticas pre profesionales son:

Actividades de aprendizaje orientadas a la aplicación de conocimientos y al desarrollo de destrezas y habilidades específicas que un estudiante debe adquirir para un adecuado desempeño en su futura profesión. Estas prácticas deberán ser de investigación-acción y se realizarán en el entorno institucional, empresarial o comunitario, público o privado, adecuado para el fortalecimiento del aprendizaje. Las prácticas pre profesionales o pasantías son parte fundamental del currículo conforme se regula en el presente Reglamento. (Consejo de Educación Superior, 2013, pág. 33)

En el mismo documento del Consejo de Educación Superior (2013, pág. 33) el cual menciona en su artículo 89 sobre las pasantías las cuales se denominan" Cuando las prácticas pre profesionales se realicen bajo relación contractual y salarial de dependencia. Serán reguladas por la normativa aplicable a las pasantías, sin modificar el carácter y los efectos académicos de las mismas.", por lo antes mencionado en cada uno de los conceptos citados en la norma regulatoria del Sistema de Educación Superior que menciona los mecanismos para que los estudiantes universitarios puedan insertarse en el mercado de trabajo los separa según las exigencias y disponibilidad de las instituciones que se ponen de acuerdo para recibir a los dicentes con el fin de que se encaminen al ámbito laboral.

Desagrega las pasantías de las practicas pre profesionales con la singularidad de que tienen una obligación contractual las pasantías, se determinaría que en base al contexto empresarial y al objeto de estudio que las prácticas profesionales son las más comunes para los dicentes en la Facultad de Ciencias Administrativas de la Universidad Técnica de Ambato.

Es por tal razón que se integra a la mayor parte de universidades las practicas pre profesionales como medio de insertar a los universitarios al contexto laboral, ya que las instituciones donde van a prepararse así lo exigen, para una mayor afluencia de personas que se formen su criterio profesional aportando de esta manera a la vinculación con la colectividad. 
En el siglo XXI se ha venido desarrollando una serie de cambios continuos y demasiado rápidos, sobre todo en los sistemas económicos que rigen en el contexto mundial, regional y local. La diversificación de la economía ha pasado de un sistema económico de mercado a un sistema económico de planificación de gobierno en menos de una década lo que anteriormente era inimaginable que estos sistemas económicos cambien repentinamente en un tiempo fugaz.

Debido a lo antes mencionado según las tendencia económicas y políticas de un gobierno, afectan a los jóvenes universitarios en su inserción laboral debido a que una forma importante de crecimiento en los países son los aportes de las industrias que muchas veces no se rigen en lo ofertan las instituciones de educación superior y empelan a profesionales de vasta experiencia para la dirección sus organizaciones, lo que influye directamente a la limitada inserción en el mercado de trabajo de los profesionales jóvenes, obligándolos a buscar empleo muchas veces en áreas que no son de su formación académica, el fenómeno del subempleo se orienta debido a la necesidad de los jóvenes en trabajar y aceptan ocupaciones para los culés no fueron orientados en su conocimiento técnico, estos empleados son sobrevalorados para sus funciones pudiendo aportar de mejor manera en los procesos laborales.

Lo que se busca en la presente investigación es hallar la herramienta idónea de inserción laboral, si se define que las personas que se encuentran empleadas y trabajando así según un horario establecido o su estabilidad laboral, además personas que no están empleadas y que trabajan bajo una serie de parámetros contractuales que por su forma de trabajo tienen ausencia en los puestos y que pueden trabajar con horarios flexibles, turnos, compensaciones, entre otros modelos de trabajo, que además se determine que se encuentre en un ámbito laboral de un empleo adecuado según a las competencias que lo determina un justo reconocimiento por su nivel de preparación universitaria, que este en un puesto ocupacional que desarrolle lo aprendido en las aulas durante mucho tiempo, eso es llegar a un nivel de empleabilidad pleno y en desarrollo, que los sectores sociales y productivos reconozcan el talento humano que ofertan las universidades. (Instituto Nacional de Encuestas y Censos, 2015)

Este fenómeno no se da en el país también es un problema a nivel regional y mundial, el cual afecta debido a los niveles de desempleo que son generados por el decrecimiento de la economía lo que se ha venido dando en estos últimos años, que nos es tan divisado como en otras épocas pero afecta, que es denominado en algunos artículos como "crisis en cámara lenta" lo cual genera impacto en la desaceleración de los mercados de trabajo en el año 2015 y que además se espera que continúe en el 2016. Los niveles de desempleo a nivel regional que alcanzo mínimos históricos del 6,2 \% en el 2014, y que aumentara a 6,7 \% en el 2016, se considera una cifra moderada si se hace un comparativo con cifras que se ha venido 
llevando por más de una década, siendo este el aumento significativo de la tasa de desocupación de los últimos años.

El desempleo en la región según los datos pasa a ser 1,7 millones de desocupados adicionales a la región lo que significa el número total de desocupados en 19 millones, la gran mayoría de estos indicadores de desocupación son mujeres y con una alta proporción jóvenes. (Organización Internacional del Trabajo , 2015, pág. 5).

Vale recalcar que así mismo la desaceleración económica no presenta datos similares en todos los países de la región debido a su alta dependencia de la economía norteamericana, en los datos a presentar creciendo a tasas más altas por efectos internacionales, en Centroamérica del 6,1 \% al 6,7 \%, el Caribe de 8,2\% a 8,55 y en México incluso referencia una baja del 5,0\% al 4,4\%. Mientras que los datos más preocupantes son los que presenta América del Sur con 6,8\% a 7,6\%, que es para el país en el cual se realiza la investigación. (Organización Internacional del Trabajo , 2015, pág. 5)

Se toma en cuenta los indicadores laborales del Ecuador con fines pertinentes para el caso de estudio, en los cuales define que la población nacional en edad de trabajar tienen un porcentaje del 69, $39 \%$ de la población total esto quiere decir que comprende a todas las personas que tienen desde 15 años o más y que en número de personas son 11,3 millones, además las personas que se encuentran en la población económicamente activa con edad de trabajar, es decir personas que se encuentran o se encontraron inmersos en el mercado laboral presentando de esta manera un 67,14\% que nos da un numero de 7,6 millones de personas que se encuentran en este indicador. Las personas que están en edad de trabajar que no tienen ningún interés por trabajar en la actualidad que generalmente son estudiantes, amas de casa, jubilados, rentistas, entre otros, conforman un 32, $96 \%$ es decir en número de personas 3,7 millones que se encuentran en la categoría mencionada. De un total de personas que se encuentran en la población económicamente activa el 95,72 \% se en cuenta empleada en las fechas de levantamiento de los datos y por lo tanto el 4,28 \% se encuentran desempleados en el país. (Instituto Nacional de Encuesta y Censos, 2015, pág. 11)

De igual manera es preocupante para los estudiantes universitarios los indicadores de subempleo debido a que la necesidad de insertarse en el mercado laboral son de suma urgencia, aceptando un cargo laboral que no es direccionado para sus competencias, en septiembre del 2015 este indicador se encontró en un 25, 82 \% disminuyéndose así desde inicio de año en 3,24 puntos porcentuales, pero que sin embargo no cumple con las obligaciones del estado para con los profesionales. (Instituto Nacional de Encuesta y Censos, 2015, pág. 14) 
Para comer en nivel de importancia de las herramientas de inserción laboral para los estudiantes de la facultad de Ciencias Administrativas de la Universidad Técnica de Ambato, es prescindible realizar una serie de encuestas que los usuarios de los servicios institucionales lo determinaran como válidos, es por eso que quienes son el objeto de estudio fueron estudiantes de Carrera de Marketing y Gestión de Negocios, en vista de que si las practicas pre profesionales son una herramienta válida que ayude a los dicentes a insertarse en el mercado laboral, que si el mecanismo antes mencionada cumple con las expectativas para desarrollar competencias que solo el ámbito profesional lo podrá orientar.

Es de suma importancia saber que los estudiantes universitarios no cuentan con experiencia ya que la mayor poparte del tiempo pasan en las Instituciones de Educación Superior con el fin captar lo expuesto en las aulas, además cumpliendo con todas las actividades exigidas por los docentes y demás autoridades que así lo disponen en su contexto universitario.

\section{Análisis e interpretación de resultados.}

Para obtener los datos se direcciono encuestas para los estudiantes de la Carrera de Marketing y Gestión de Negocios de la Universidad Técnica de Ambato con un total de 333 estudiantes de séptimo a decimo semestre de la carrera ya que ellos son quienes pueden realizar sus prácticas profesionales, debido a que ya cumplen con un perfil de las asignaturas profesionalizantes que requieren para desenvolverse en el mercado de trabajo, se realiza un muestreo estratificado y aleatorio para obtener una muestra representativa del objeto de estudio trabajando con un 95\% de aceptabilidad que es lo frecuente en los estudios de investigación de la Ciencias Sociales, obteniendo un total de 188 estudiantes los cuales tienen que ser encuestados en forma aleatoria y probabilística para no sesgar los datos que se desean obtener.

\section{Preguntas. Caracterización de la muestra estudiada}

El nivel de los conocimientos que se obtiene en la carrera universitaria a lo largo de su malla curricular según lo que menciona la muestra encuestada, se determina que solo el 7, $69 \%$ de los estudiantes consideran que los conocimientos obtenidos en su vida universitaria son excelentes, lo que determina la necesidad de reforzar la calidad de la enseñanza para que los estudiantes universitarios puedan tener oportunidades en el mercado laboral, que por consiguiente se debería fortalecer la rigurosidad y la forma de dictar clases por parte de los docentes de la institución con el fin de cumplir con las expectativas de los estudiantes. 
Gráfica 1. Calidad de los conocimientos que se obtienen.

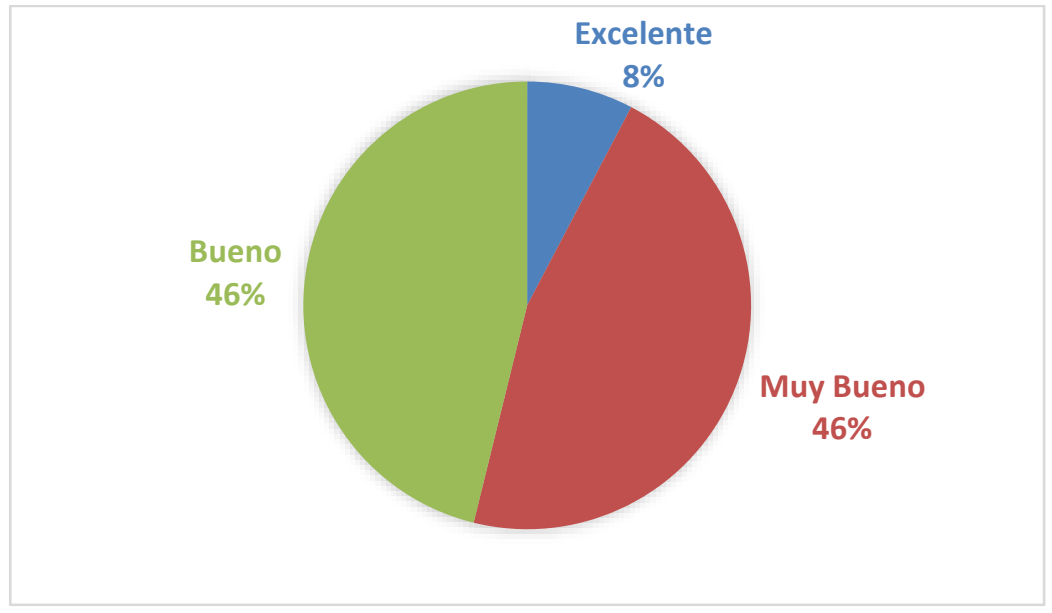

Elaborado por: equipo investigador.

En relación a las encuestas realizadas se define que solo el 9,9 \% de los estudiantes consideran que sus destrezas y habilidades profesionales han sido desarrolladas en las aulas, siendo este un indicador no tan favorecedor para la gestión académica de la institución, el $62,09 \%$ de igual manera manifiesta que sus competencias son medianamente adecuada pero no cumplen con las expectativas deseadas para el mercado de trabajo, por lo cual es determinante realizar simulaciones que permitan desarrollar habilidades y destrezas que permitan incrementar ciertas competencias en los estudiantes.

Gráfica 2. Fortalecimiento de habilidades y destrezas.

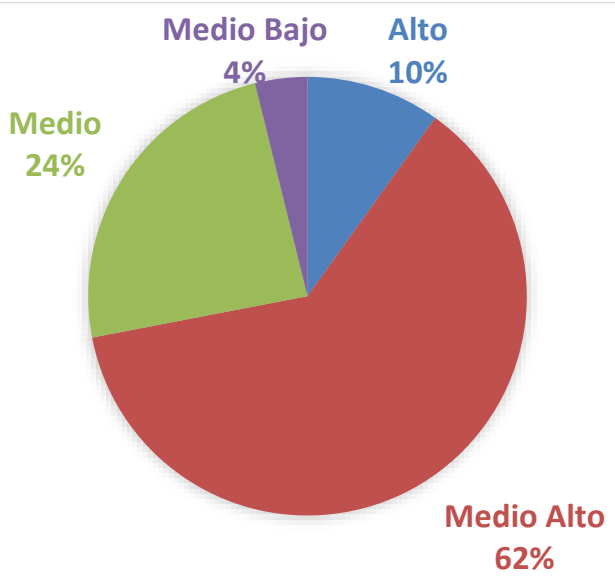

Elaborado por: equipo investigador. 
Dentro de las necesidades planteadas son consideradas las herramientas administrativas como una forma brindar solución a los problemas en el en contexto laboral, que amerita de forma técnica la metodología de Ciencias Administrativas, el 53,8\% de los encuestados no tienen en su totalidad conocimientos de herramientas administrativas para la solución de problemas, siendo este el porcentaje más elevado, determina el nivel no eficiente en brindar casos prácticos que permitan obtener conocimientos de herramientas claves para que el estudiante pueda desenvolverse en el mercado laboral.

Gráfica 3. Conocimientos suficientes.

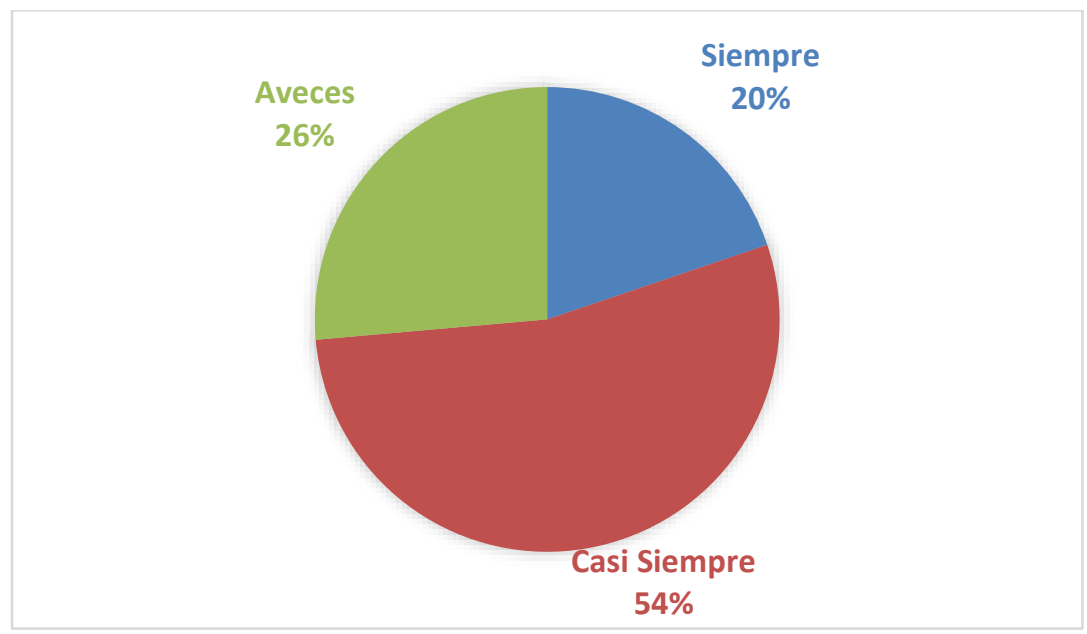

Elaborado por: equipo investigador.

De los resultados obtenidos podemos interpretar que en su mayoría no tienen una enseñanza optima de las competencias aptitudinales de igual manera no se llega a un nivel óptimo y en ciertos casos es regular con un nivel del 2,2\%, lo cual determina que hay una población que no está satisfecha con la formación de sus competencias, y el 12,1\% que se sienten conformes con su formación, el resto de la población se encuentra conforme , pero eso no quiere decir que se encuentre en su mejor desarrollo, por lo que se debe estar pendiente y encaminar a las aptitudes de los estudiantes para su desenvolvimiento en el mercado laboral. 
Gráfica 4. Competencias en el ámbito laboral.

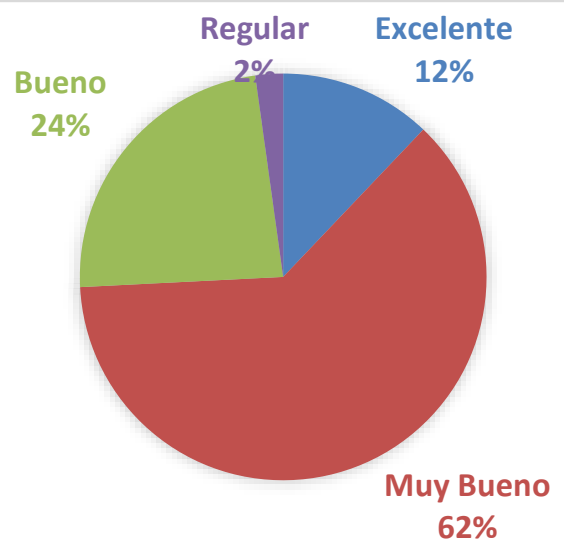

Elaborado por: equipo investigador.

El mayor porcentaje de la muestra encuestada manifiesta que en el manejo de situaciones laborales su respuesta es mínima en relación a las prácticas pre profesionales, aun siendo que el $22 \%$ de los encuestados definen a las practicas pre profesionales como mecanismo idóneo para la inserción en el mercado de trabajo, es por el motivo de que los estudiantes universitarios en su mayoría de veces no realizan funciones encaminadas a su perfil profesional, los niveles de compromiso con los practicantes en las empresas que se encuentran realizando este requisito, no aportan, ya que no tienen un seguimiento, políticas y gestión necesaria que permita en tomar correctivos en este ámbito, por lo cual tener un mínimo porcentaje del $12 \%$ de estudiantes que consideran que las practicas pre profesionales son aporte en su ámbito laboral, demuestra que hay que tomar correctivos y mejorar los mecanismos de inserción en el mercado de trabajo para los estudiantes universitarios.

Gráfica 5. Aporte de las prácticas pre profesionales en el ámbito laboral.

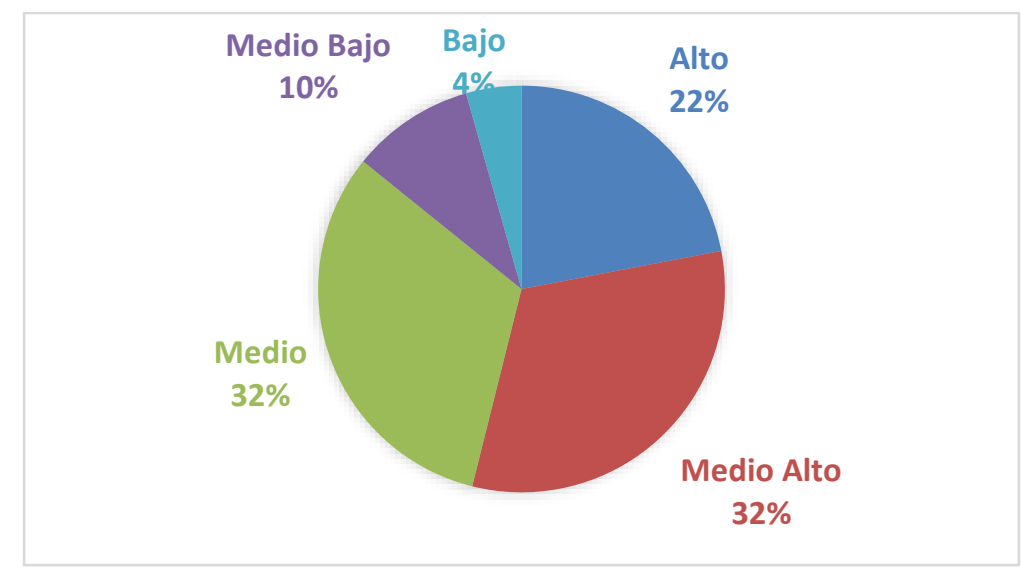

Elaborado por: equipo investigador. 
En referencia a las investigación realizada se acota que las instituciones de educación superior están encaminada a obtener resultados de la inserción laboral de los estudiantes universitarios que se graduaron a hace un periodo anterior, por lo cual se enfocan en obtener resultados de los estudiantes que ya se graduaron para justificar la pertinencia de las carreras universitarias en el contexto, para lo cual la importancia de las universidades debe ser enfocada, en formación, carreras y mallas curriculares que ofertan sean necesarias para tener profesionales de éxito, por lo tanto, las instituciones de educación superior deben enfocarse en los dicentes para determinar en qué van a trabajar a futuro, desde que se inicia una carrera universitaria, la formación en los periodos de educación, y la consecución de un puesto de trabajo, acorde a las formación profesional que obtiene el dicente (Arteaga Briones, Capó Pérez, \& Ruiz Arnaud, 2017), González Jaimes \& Miguel Silva (2017) los conceptos y enseñanzas en la educación superior no son óptimas, la educación que reciben los estudiantes universitarios no son suficientes para desenvolverse en el mercado laboral, los empresarios necesitan que los profesionales que egresan recién de la universidad, estén aptos para tomar decisiones y resolver problemas en las áreas de mayor impacto en las organizaciones, por lo cual la responsabilidad de incrementar y generar nuevos mecanismos que ayuden al dicente a desarrollar las competencias laborales por medio de simulación de casos prácticos y técnicos en las áreas específicas de estudio, por otro lado, los estudiantes universitarios no son más que la proyección de las instituciones de educación superior, por lo cual, es de suma importancia que se tome en consideración las necesidades de las instituciones públicas y privadas, con el fin de dar respuestas agiles a la sociedad, de igual forma la creciente competencia de profesionales extranjeros que por su condición en su país de origen no tiene costos elevados y en ciertos casos la formación profesional es gratuita, estos tienen mayor competencia en el perfil duro (formación universitaria), esto limita las posibilidades de que los profesionales universitarios de nuestro contexto que no tienen las facilidades antes mencionadas, obtengan más oportunidades de conseguir empleo (Rodríguez Garcés \& Padilla Fuentes, 2017), es por tal motivo que las instituciones de educación superior deben tener como principal objetivo ofertar carreras universitarias que estén en vigencia de las necesidades de la sociedad en general.

Carvajal, Romero, \& Alvaréz (2017) en su investigación mencionas que la formación universitaria debe ser teórica y practica alavés, por lo cual incrementar el número de horas de participación de los estudiantes en las organizaciones es de suma importancia para incrementar las competencias de los dicentes lo daría mayores competencias laborales, siendo profesionales atractivos para los empresarios e instituciones públicas.

De la misma forma se determina que las universidades son el eje principal para la generación de oportunidades laborales de sus estudiantes, por un razón lógica, ofertar carreras que estén ancladas a las necesidades de la sociedad y que tengan pertinencia con las necesidades de la sociedad (Tamayo Maggi \& Cerda Paredes, 2017), en relación a lo expuesto el equipo 
investigador concuerda con las teorías expuestas, por lo cual es necesario que se determinen nuevos mecanismos de formación profesional para los estudiantes universitarios.

\section{Conclusiones.}

- El nivel de empleabilidad en América Latina es dependiente de los indicadores macroeconómicos de la región y países desarrollados, por lo cual es necesario determinar cuáles son las necesidades del contexto en el cual se desenvuelven para generar oportunidades de trabajo para los dicentes universitarios.

- Al estar deficiente los indicadores de formación profesional de los estudiantes de la Carrera de Marketing y Gestión de Negocios de la Facultad de Ciencias Administrativas de la UTA, es necesario realizar un diagnóstico situacional en general para determinar los mejores perfiles docentes que puedan aportar con experiencia profesional en la formación del dicente.

- Es de suma importancia generar nuevos mecanismos de formación profesional para que interactúen los estudiantes de la Carrera de Marketing y Gestión de Negocios de la Facultad de Ciencias Administrativas de la UTA con las organizaciones públicas y privadas, para que en el día conozcan de las necesidades del contexto.

- Contar con docentes en la Carrera de Marketing y Gestión de Negocios de la Facultad de Ciencias Administrativas de la UTA que tengan experiencia empresarial en el área, ya que según las encestas realizadas en su gran mayoría la formación de los estudiantes son solamente teóricas sin tener experiencias profesionales, y al ser una carrera técnica es necesario tener profesores que por medio de la experiencia profesional puedan formar profesionales capaces de resolver problemas en la áreas específicas.

\section{Referencias bibliográficas.}

Arteaga Briones, L. A., Capó Pérez, J. R., \& Ruiz Arnaud, J. R. (2017). El seguimiento de graduados: una perspectiva desde los docentes y estudiantes de la carrera de Medicina Veterinaria y Zootecnia de la Universidad Técnica de Manabí. Revista cubana de educación superiror, 67-75.

Asamblea Nacional Cosntituyente de la Republica del Ecuador. (12 de Octubre de 2010). Ley Órganica de Educación Superior. San Fransisco de Quito, Pichincha, Ecuador: Lexis S.A.

Carvajal, R., Romero, A., \& Alvaréz, G. (2017). Estrategia para Contribuir a la Implementación de la Formación Dual de los Profesionales de Ciencias 
Empresariales en las Pequeñas y Medianas Empresas de la Provincia Tungurahua, Ecuador. Formación universitaria, 29-41.

Consejo de Educación Superior. (21 de Noviembre de 2013). Reglamento de Régimen Académico. San Fransisco de Quito, Pichincha, Ecuador: Consejo de Educación Superior.

García Blanco, M., \& Cárdenas Sempértegui, E. (2016). La inserción laborar en la educación superior. La perspectiva latinoamericana. Educación XX1, 2-28.

González Jaimes, E. I., \& Miguel Silva, M. G. (2017). Egresados universitarios y su pertinencia para el ingreso a la empleabilidad empresarial o la autoempreabilidad. Revista Electrónica de Psicología Iztacala, 168-185.

Instituto Nacional de Encuesta y Censos. (2015). Encuesta Nacional de Empleo, Desempleo y Subempleo. San Fransisco de Quito: Instituto Nacional de Encuestas y Censos.

Instituto Nacional de Encuestas y Censos. (2015). Indicadores Laborales Junio 2015. San Fransisco de Quito: Instituto Nacional de Encuestas y Censos.

Organización Internacional del Trabajo . (2015). Panorama Laboral 2015. Lima: Oficina Regional para América Latina y el Caribe.

Rodríguez Garcés , C., \& Padilla Fuentes, G. (2017). Formación profesional y empleabilidad: las tensiones del mercado en las carreras de la salud en Chile. Educación Médica Superior, 1-9.

Tamayo Maggi, M., \& Cerda Paredes, M. (2017). Rol de la pertinencia en la evaluación de carreras universitrias en el Ecuador. Revista Ciencias Pedagógicas e Innovación, 19.

Vacchiano, M., Martí, J., Yepes , L., \& Miquel , J. (2018). Las redes personales en la inserción laboral juvenil en tiempos de crisis. Un análisis en Barcelona. Reis. Rev.Esp.Investig.Social, 121-140.

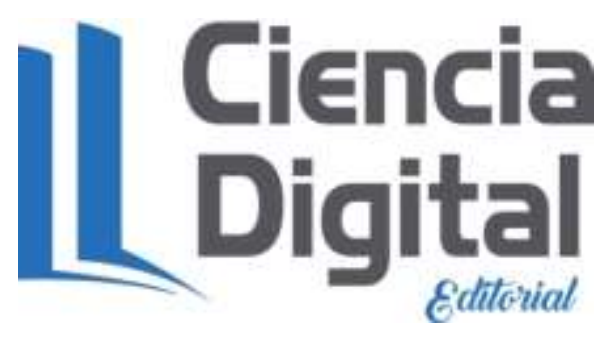


Para citar el artículo indexado.

Salazar L., Plaza J., Velastegui E. Salazar L. \& Carrasco T. (2018). Componente laboral y su inserción en el mercado de trabajo de los graduados de la carrera de marketing y gestión de negocios. Revista electrónica Ciencia Digital 2(1), 141-153. Recuperado desde: http://www.cienciadigital.org/revistascienciadigital/index.php/CienciaDigital/article/view/1 $0 / 10$

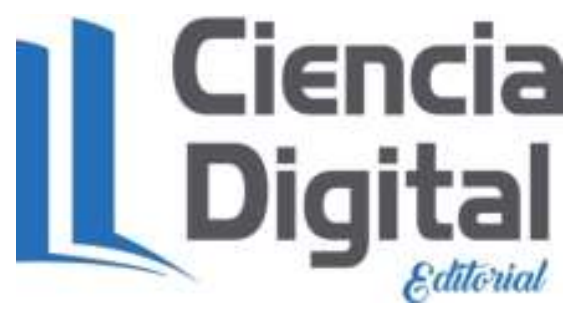

El artículo que se publica es de exclusiva responsabilidad de los autores y no necesariamente reflejan el pensamiento de la Revista Ciencia Digital.

El articulo queda en propiedad de la revista y, por tanto, su publicación parcial y/o total en otro medio tiene que ser autorizado por el director de la Revista Ciencia Digital.
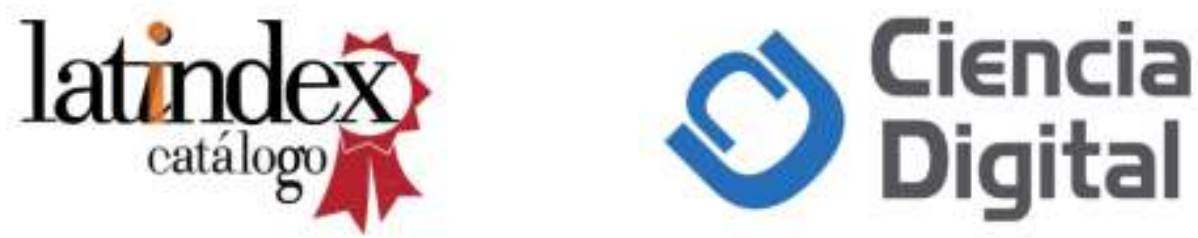